\title{
Protective effect of taurine on sepsis-induced lung injury via inhibiting the p38/MAPK signaling pathway
}

\author{
JIAO CHEN $^{1 *}$, XIANG XUE $^{1 *}$, JIANQIN CAI $^{1}$, LING JIA $^{1}$, BAODI SUN $^{2}$ and WEI ZHAO ${ }^{1}$ \\ Departments of ${ }^{1}$ Critical Care Medicine and ${ }^{2}$ Emergency Medicine, Sir Run Run Hospital, \\ Nanjing Medical University, Nanjing, Jiangsu 211100, P.R. China
}

Received August 6, 2020; Accepted June 9, 2021

DOI: $10.3892 / \mathrm{mmr} .2021 .12292$

\begin{abstract}
Sepsis, a leading cause of acute lung injury (ALI), is characterized by an overwhelming systemic inflammatory response and widespread organ injury, particularly in the lungs. Taurine, an intracellular free amino acid, has been used for the treatment of various diseases, including lung injury; however, the underlying mechanisms are unclear. The present study aimed to investigate the protective effect of taurine on septic ALI and the underlying mechanism. A septic ALI model was established by performing cecal ligation and puncture (CLP) surgery on Sprague Dawley rats. Following successful model establishment, rats were treated with taurine. The results of hematoxylin and eosin, respiratory function detection, malondialdehyde level and superoxide dismutase activity determination and ELSIA demonstrated that taurine significantly alleviated lung injury, restored respiratory function, reduced oxidation and decreased the concentrations of inflammatory factors in CLP-induced septic ALI model rats. In addition, compared with that in the ALI group, western blotting results indicated that taurine ameliorated lung epithelial injury by significantly increasing the expression levels of lung epithelial markers, E-cadherin and occludin. The western blotting results demonstrated that, compared with the control group, the $\mathrm{p} 38 / \mathrm{MAPK}$ and NF- $\mathrm{B}$ signaling pathways were
\end{abstract}

Correspondence to: Professor Wei Zhao, Department of Critical Care Medicine, Sir Run Run Hospital, Nanjing Medical University, 109 Longmian Road, Nanjing, Jiangsu 211100, P.R. China

E-mail: 13605158009@163.com

Dr Baodi Sun, Department of Emergency Medicine, Sir Run Run Hospital, Nanjing Medical University, 109 Longmian Road, Nanjing, Jiangsu 211100, P.R. China

E-mail: sunbaodi321@163.com

${ }^{*}$ Contributed equally

Abbreviations: ALI, acute lung injury; CLP, cecal ligation and puncture; ARDS, acute respiratory distress syndrome; MDA, malondialdehyde; SOD, superoxide dismutase

Key words: sepsis, ALI, taurine, p38/MAPK signaling, SB203580 significantly activated in CLP-induced septic ALI model rats, but taurine significantly suppressed ALI-mediated signaling pathway activation. To investigate the mechanism underlying taurine in the treatment of septic ALI, CLP-induced septic ALI model rats were treated with an antagonist of the p38/MAPK signaling pathway (SB203580). The effects of SB203580 on CLP-induced septic ALI model rats were similar to those of taurine. SB203580 significantly attenuated sepsis-induced lung injury and increases in IL-1 $\beta$ and TNF- $\alpha$ concentrations in the lung tissue. In addition, SB203580 promoted restoration of the injured lung tissue and respiratory function in CLP-induced septic ALI model rats. The western blotting results indicated that SB203580 significantly decreased the ratios of phosphorylated (p)-p38/p38 and p-p65/065, and increased the protein expression levels of E-cadherin and occludin compared with those in the ALI group. In summary, the present study demonstrated that taurine alleviated sepsis-induced lung injury, which was associated with suppression of the inflammatory response and oxidative stress via inhibiting the p38/MAPK signaling pathway. Therefore, the p38/MAPK signaling pathway may serve as a potential therapeutic target for the treatment of sepsis-induced ALI.

\section{Introduction}

Sepsis results in life-threatening organ dysfunction that is characterized by overwhelming systemic inflammation (1). Lung inflammation, as a type of acute lung injury (ALI), is often induced in severe sepsis (2). Despite improvements in intensive care management and multidisciplinary treatments, the mortality rate of sepsis was $17 \%$ and was $26 \%$ for severe sepsis during 1979-2015 worldwide (3-7). Therefore, the pathogenesis of sepsis-induced lung injury requires further investigation to identify new effective therapeutics.

Previous studies have indicated that sepsis-induced lung injury is primarily associated with systemic inflammatory response syndrome, including the activation of inflammatory cytokines and inflammatory signaling pathways (8-10). Numerous inflammatory signaling pathways serve an important role in the pathogenesis of sepsis-induced lung injury, including the MAPK and NF- $\mathrm{B}$ signaling pathways. The MAPK and NF- $\kappa \mathrm{B}$ signaling pathways induce the excessive release of inflammatory cytokines, including TNF- $\alpha$, IL-1 $\beta$ and other associated cytokines, which contribute to lung 
dysfunction and mortality in sepsis-induced lung injury (11-13). p38/MAPK is a key member of the MAPK signaling pathway that serves an essential role in cell proliferation and survival, and can be activated by various inflammatory mediators and environmental stress (14). Previous studies have reported that activation of the $\mathrm{p} 38 / \mathrm{MAPK}$ signaling pathway is involved in the development of ALI/acute respiratory distress syndrome (ARDS). These studies indicated that pharmacological targeting of the p38/MAPK signaling pathway could decrease the expression levels of inflammation factors and attenuate ALI/ARDS $(15,16)$.

Taurine (2-aminoethanesulfonic acid) is a type of intracellular free amino acid. As a cysteine derivative, taurine is highly expressed in all mammalian tissues and has important physiological effects on all cells in the body, including endogenous antioxidant, detoxification, osmoregulation, anti-inflammatory and membrane stability effects (17-19). In addition, previous studies have indicated that taurine could protect against lung injury by modulating oxidative stress, reducing cell apoptosis and decreasing the expression levels of inflammatory factors (20-22). Therefore, taurine may serve as a novel candidate for treating sepsis-induced ALI; however, the underlying molecular mechanism remains unclear.

The aim of the present study was to investigate the protective effects of taurine on sepsis-induced ALI, as well as the underlying molecular mechanism. In particular, the effects of taurine on lung tissue pathological changes, respiratory function, malondialdehyde (MDA) levels, superoxide dismutase (SOD) activity and inflammatory factor concentrations were evaluated in a Sprague Dawley (SD) cecal ligation and puncture (CLP)-induced septic ALI rat model. Furthermore, the roles of the p38/MAPK and NF- $\kappa B$ signaling pathways in the pathogenesis of sepsis-induced ALI were investigated.

\section{Materials and methods}

Ethics statement. The present study was conducted according to the Guide for the Care and Use of Experimental Animals established by the National Society for Medical Research (23). The present study was approved by the Ethics Committee of Nanjing Medical University (approval no. SYXK 2019-0042). All surgeries on the rats were conducted under anesthesia (sodium pentobarbital), and every effort was made to alleviate pain.

Animal model and study design. In total, 168 SD rats (male; age, 8-10 weeks; weight, 220-250 g) were provided by the Laboratory Animal Centre of Nanjing Medical University. All rats were acclimatized for 7 days prior to the experiment, fed with standard chow (Global Diet; Shanghai, China) and provided with ad libitum access to water in standard shoebox cages. The rats were housed in a normal atmosphere under 12-h light/dark cycles at $20-25^{\circ} \mathrm{C}$ with $50-60 \%$ humidity. The surgical procedure to establish the CLP-induced septic rat model was performed as previously described (24). Briefly, all rats were fasted and anesthetized by the intraperitoneal injection of $50 \mathrm{mg} / \mathrm{kg}$ sodium pentobarbital (Sigma-Aldrich; Merck KGaA). Subsequently, the middle abdominal incision site was shaved and the cecum was exposed via a $2-\mathrm{cm}$ abdominal midline incision. The cecum was mobilized, ligated and punctured with a 21-gauge needle. Some of the fecal matter was squeezed through the puncture wound, then the bowel was repositioned and the abdomen was sutured. The animals were resuscitated with a subcutaneous sterile saline immediately after CLP surgery. The sham-operated control rats underwent the same procedure, but without ligation or puncture of the cecum. No mortality was observed in the rats after CLP was induced. At 3 days post-CLP, rats were anesthetized by the intraperitoneal injection of $50 \mathrm{mg} / \mathrm{kg}$ sodium pentobarbital (Sigma-Aldrich; Merck KGaA) and blood samples were collected via cardiac puncture using a $1.5 \mathrm{ml}$ tube. Subsequently, the anesthetized rats were sacrificed by cervical dislocation. The lung tissue samples were collected for further analysis. Death was confirmed by monitoring cessation of the heartbeat and pupil dilation.

To investigate the protective effects of taurine on sepsis-induced lung injury, SD rats were randomly assigned to the following three groups ( $n=24$ per group): i) Control, rats received an intraperitoneal injection of normal saline $(1 \mathrm{ml} / \mathrm{kg} ; 200 \mu \mathrm{l})$; ii) ALI, CLP-induced septic ALI model rats received an intraperitoneal injection of normal saline $(1 \mathrm{ml} / \mathrm{kg} ; 200 \mu \mathrm{l})$; iii) ALI + taurine, CLP-induced septic ALI model rats received an intraperitoneal injection of taurine for 3 days $(200 \mathrm{mg} / \mathrm{kg} / \mathrm{day}$ in $200 \mu \mathrm{l}$ normal saline; Sigma-Aldrich; Merck KGaA). To clarify the molecular mechanisms underlying taurine-mediated protection of lung tissue following CLP induction, rats were randomly assigned to the following four groups ( $\mathrm{n}=24$ per group): i) Control, rats received an intraperitoneal injection of normal saline $(1 \mathrm{ml} / \mathrm{kg}$; $200 \mu \mathrm{l})$; ii) ALI, CLP-induced septic ALI model rats received an intraperitoneal injection of normal saline $(1 \mathrm{ml} / \mathrm{kg} ; 200 \mu \mathrm{l})$; iii) ALI + taurine, CLP-induced septic ALI model rats received an intraperitoneal injection of taurine for 3 days $(200 \mathrm{mg} / \mathrm{kg} / \mathrm{day}$ in $200 \mu \mathrm{l}$ normal saline; Sigma-Aldrich; Merck KGaA); and iv) ALI + SB203580, CLP-induced septic ALI model rats received an intraperitoneal injection of SB203580 (10 mg/kg in $200 \mu 1$ normal saline; Sigma-Aldrich; Merck KGaA). The dose selection and route of administration of taurine was based on our previous study (14).

Respiratory function detection. Prior to sacrifice, arterial blood gas content was examined using i-STAT ${ }^{\circledR}$ G7+ cartridges and an i-STAT portable clinical analyzer (Abbott Point of Care Inc.). The cartridges were recovered immediately before use to avoid compression. For arterial blood gas analysis, arterial blood gas was sampled from the left ventricle of each rat, added into the sample well and allowed to fill by passive movement to the indicated level (volume, 80-100 ml). The cap on the sample well was closed and the cartridge was inserted into the analyzer. The values of partial pressure $(\mathrm{Pa})$ of oxygen $\left(\mathrm{PaO}_{2}\right)$ and carbon dioxide $\left(\mathrm{PaCO}_{2}\right)$ were recorded after the calibration and analysis cycle.

ELISA. Part of right lung tissue was cut into pieces and mechanically disrupted into a homogenate with RIPA buffer (Beyotime Institute of Biotechnology) containing protease inhibitor cocktail (Roche Diagnostics $\mathrm{GmbH}$ ). Then, the samples were centrifuged at $12,000 \mathrm{x}$ g for $30 \mathrm{~min}$ at $4^{\circ} \mathrm{C}$ 
and the supernatants were collected. The concentrations of IL-1 $\beta$ and TNF- $\alpha$ in the supernatants were measured using commercially available ELISA kits according to the manufacturer's instructions. The IL-1 $\beta$ (cat. no. ELR-IL1b) and TNF- $\alpha$ (cat. no. ELR-TNFa1) ELISA kits were purchased from Ray Biotech, Inc.

MDA level and SOD activity detection. The level of MDA is an index of membrane lipid peroxidation, and its level reflects the lipid peroxidation rate and strength. Moreover, MDA levels can be used as a marker to indicate the degree of tissue peroxidation damage. SOD serves an important role in the balance of oxidation and antioxidation. SOD can remove superoxide anion free radicals and protect cells from damage (25). The activity of SOD and the levels of MDA were measured in the lung tissue sample supernatants as previously described (25). The SOD (cat. no. A001-1-2) and MDA (cat. no. A003-1-1) kits were purchased from Nanjing Jiancheng Bioengineering Institute, and were used according to the manufacturer's protocol. Briefly, the right lung tissue of each rat was dissected and homogenized with three volumes of ice-cold $1.15 \%$ potassium chloride (ratio of tissue to potassium chloride was 1:3). To determine MDA levels, the reaction mixture $(0.2 \mathrm{ml}$ $8.0 \% \mathrm{SDS}, 1.5 \mathrm{ml} 20 \%$ acetic acid and $1.5 \mathrm{ml} 0.8 \%$ aqueous thiobarbituric acid) was added to $0.1 \mathrm{ml}$ homogenized tissue and adjusted to $\mathrm{pH}$ 3.5. Then, the final volume was adjusted to $4.0 \mathrm{ml}$ with distilled water. Subsequently, $5.0 \mathrm{ml} \mathrm{N}$-butanol and pyridine mixture [15:1 (v/v)] was added. The mixture was shaken vigorously and centrifuged at $1,200 \mathrm{x} \mathrm{g}$ for $10 \mathrm{~min}$ at $4^{\circ} \mathrm{C}$. Finally, the absorbance of the organic layer was measured at a wavelength of $532 \mathrm{~nm}$ using a Spectronic UV 120 spectrophotometer (Thermo Fisher Scientific, Inc.). The MDA levels are presented as nmol/mg protein.

Xanthine and xanthine oxidase are used to generate superoxide radicals that react with $\mathrm{p}$-iodonitrotetrazlium violet (INT) to form a red formazan dye, which can be measured with a Spectronic UV 120 spectrophotometer (Thermo Fisher Scientific, Inc.) at a wavelength of $505 \mathrm{~nm}$ to determine SOD activity (26). SOD activity is presented as $\mathrm{U} / \mathrm{mg}$ protein. The assay mixture contained $0.01 \mathrm{M}$ phosphate buffer, 3-cyclohexilamino-1-propanesulfonic acid (CAPS) buffer solution (50 mM CAPS and $0.94 \mathrm{mM}$ EDTA in a saturated solution of sodium hydroxide; $\mathrm{pH} 10.2)$, substrate solution $(0.05 \mathrm{mM}$ xanthine and $0.025 \mathrm{mM}$ INT) and $80 \mu \mathrm{l}$ xanthine oxidase.

Histological examination. After fixing with $4 \%$ neutral phosphate-buffered paraformaldehyde for $16 \mathrm{~h}$ at room temperature, the lower left lung was embedded in paraffin and sectioned into $5-\mu \mathrm{m}$ thick slices. To determine the integrity of the histology structure and the collagen deposition condition, the slices were stained with hematoxylin and eosin (H\&E; Beyotime Institute of Biotechnology) according to the manufacturer's instructions. Stained sections were observed using a light microscope (Olympus Corporation) and scored by two pathologists who were blinded to the samples and had expertise in lung pathology. The lung injury score was determined using the Tanino Method (27). In brief, morphological alterations in the lungs were scored based on the extent of pathology on a scale of $0-4$ by assessing interstitial edema, alveolar edema, hemorrhage and neutrophil infiltration. The following scoring values were used: 0 , no reaction in the alveolar walls; 1, diffuse reaction in the alveolar walls, but without thickening of the interstitium; 2, diffuse presence of the inflammatory cells in the alveolar walls with a slight thickening of the interstitium; 3, moderate interstitial thickening accompanied by inflammatory cell infiltrates; and 4 , interstitial thickening involving $>1 / 2$ of the microscopic field. The results are expressed as the average of the values from 30 microscopic fields.

Lung wet/dry ratio determination. After sacrifice, the left upper lung tissue $(\sim 1 \mathrm{~g})$ was dissected, cleansed of blood or liquid with absorbent paper, weighed to obtain the 'wet' weight and then heated in an $80^{\circ} \mathrm{C}$ thermostatic oven for $48 \mathrm{~h}$. Subsequently, the tissue was weighed again to obtain the 'dry' weight. The ratio of wet to dry lung was calculated to assess tissue edema.

Western blotting. Western blotting was performed as previously described (28). Proteins were extracted from lung tissues using RIPA (Beyotime Institute of Biotechnology) containing protease inhibitor cocktail (Roche Diagnostics $\mathrm{GmbH}$ ) for $30 \mathrm{~min}$ on ice. Protein concentrations were determined using the BCA Protein Assay Kit (Beyotime Institute of Biotechnology). All the protein samples were boiled for 10 min in equal volumes of 5X SDS buffer. Subsequently, proteins $(20 \mu \mathrm{g})$ were separated via $12 \%$ SDS-PAGE and transferred to PVDF membranes (Roche Diagnostics $\mathrm{GmbH}$ ) using a semi-dry transfer apparatus (Bio-Rad Laboratories, Inc.). After blocking with 5\% skimmed milk in PBS-Tween-20 (PBST; PBS with 0.5\% Tween-20) at room temperature for $1 \mathrm{~h}$ and washing three times with PBST, the membranes were incubated overnight at $4{ }^{\circ} \mathrm{C}$ with the following primary antibodies (all purchased from Abcam): Rabbit anti-occludin (1:1,000; cat. no. ab216327), rabbit anti-E-cadherin (1:10,000; cat. no. ab76319), rabbit anti-p-p65 (1:1,000; cat. no. ab194726), rabbit anti-p65 (1:1,000; cat. no. ab16502), rabbit anti-p-p38 (1:1,000; cat. no. ab47363), rabbit anti-p38 (1:3,000; cat. no. ab170099) and mouse anti- $\beta$-actin (1:3,000; cat. no. ab7817). After three washes with PBST, the membranes were incubated with a horseradish peroxidase-conjugated goat anti-rabbit or anti-mouse secondary antibody (1:5,000; cat. nos. BM2006 or BA1050, respectively; Wuhan Boster Biological Technology, Ltd.) for $1 \mathrm{~h}$ at $37^{\circ} \mathrm{C}$. Immunocomplexes were visualized using an enhanced chemiluminescence system (Thermo Fisher Scientific, Inc.) and an Odyssey Scanning System (LI-COR Biosciences). Protein expression was semi-quantified using Image J software (version 3.0; National Institutes of Health) with $\beta$-actin as the loading control.

Statistical analysis. All experiments were repeated at least three times with nearly identical results. Statistical analyses were performed using SPSS software (version 21.0; IBM Corp.). Data are presented as the mean \pm standard deviation. Parametric data were analyzed using one-way ANOVA followed by Tukey's post hoc test. Data with a non-parametric distribution were analyzed using the Kruskal-Wallis test followed by Dunn's post hoc test. $\mathrm{P}<0.05$ was considered to indicate a significant difference. 
A

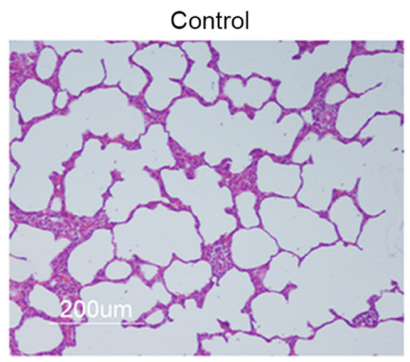

B

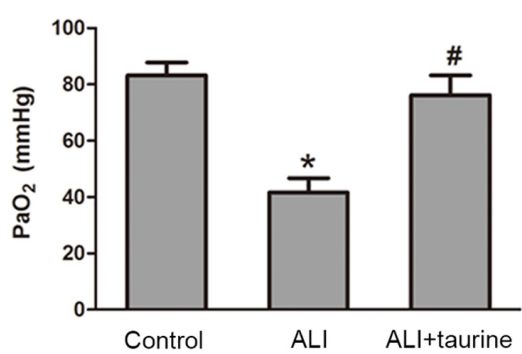

D

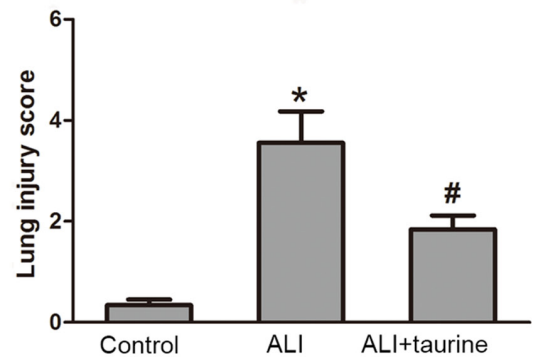

ALI

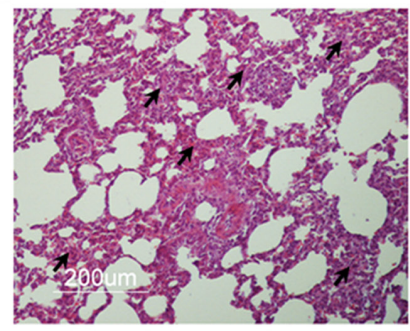

C

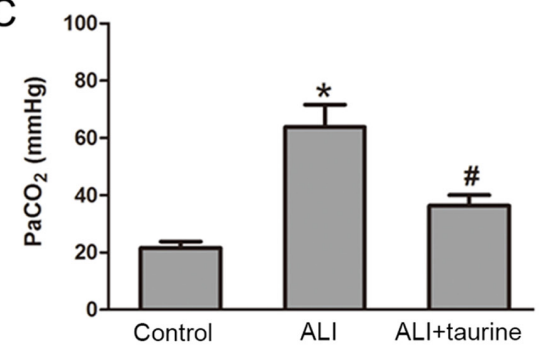

$\mathrm{E}$

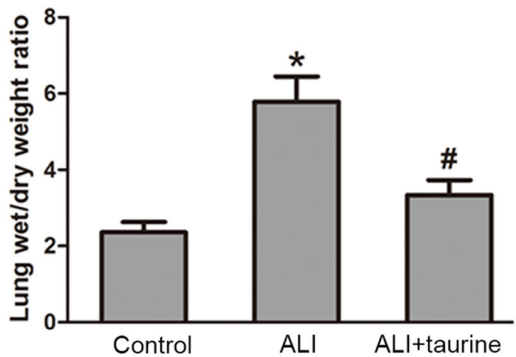

Figure 1. Taurine suppressed lung injury in CLP-induced septic ALI model rats. Acute lung injury was induced in rats by sepsis. At day 3 post-CLP, analyses were performed. CLP-induced septic ALI model rats were treated with or without taurine, and then lung tissues in each group were analyzed by performing H\&E staining, respiratory function detection tests and lung injury score examinations. (A) Representative images of H\&E staining in each group (magnification, x100). Black arrows indicate neutrophil infiltration. Content of (B) $\mathrm{O}_{2}$ and (C) $\mathrm{CO}_{2}$ in arterial blood. (D) Lung injury score in each group. (E) Lung wet/dry weight ratio of the lung tissues in each group. Data are presented as the mean \pm standard deviation. ${ }^{*} \mathrm{P}<0.05$ vs. control; ${ }^{*} \mathrm{P}<0.05$ vs. ALI. CLP, cecal ligation and puncture; H\&E, hematoxylin and eosin; Pa, partial pressure; ALI, acute lung injury.

\section{Results}

Taurine suppresses sepsis-induced ALI in rats. The lung is particularly susceptible to acute injury in CLP-induced polymicrobial sepsis (29). To determine the protective effect of taurine on sepsis-induced lung injury, a series of lung sections obtained from the rats in each group were stained with H\&E (Fig. 1A). The tissue sections obtained from the control rats were histologically normal and displayed no inflammation or epithelial damage. After CLP-induced sepsis, the lung tissue sections from rats in the ALI group displayed extensive cellular thickening of the interalveolar septa and interstitial edema, and infiltration of inflammatory cells. However, inflammatory cell infiltration and edema were notably ameliorated in the lungs following treatment with taurine in ALI rats. As shown in Fig. 1B and C, the respiratory function test results indicated that $\mathrm{PaO}_{2}$ levels in the ALI group were significantly decreased, but $\mathrm{PaCO}_{2}$ levels were significantly increased compared with those in the control group. Taurine treatment significantly increased $\mathrm{PaO}_{2}$ levels and decreased $\mathrm{PaCO}_{2}$ levels in CLP-induced septic ALI model rats. The lung injury score was also evaluated using histological sections and a semi-quantitative scale (Fig. 1D). The lung injury score following CLP was significantly increased compared with that in the control group, whereas the score was significantly decreased in CLP-induced septic ALI model rats following treatment with taurine. In addition, after lung injury, sepsis resulted in an increase in lung weight owing to inflammatory and fibrotic components. Compared with that in the control group, the lung wet/dry ratio in the ALI group was significantly increased (Fig. 1E). By contrast, the lung wet/dry ratio was significantly decreased in the ALI + taurine group compared with that in the ALI group. These results indicated that taurine protected the lung tissue and attenuated sepsis-induced injury.

MDA levels and SOD activity in lung tissues following taurine treatment. MDA levels and SOD activity in the lung tissues of each group were detected to assess the effects of taurine on oxidative stress. A significant increase in MDA levels (Fig. 2A) and a decrease in SOD activity (Fig. 2B) was observed in the lung tissue of the ALI group compared with that of the control group. The results indicated that sepsis induced membrane lipid peroxidation and superoxide toxicity in the lung tissue, whereas taurine treatment significantly reversed ALI-mediated effects, suggesting that taurine may serve a protective role against oxidative stress. 
A

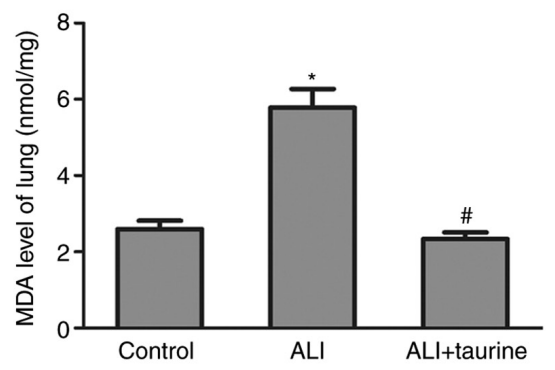

C

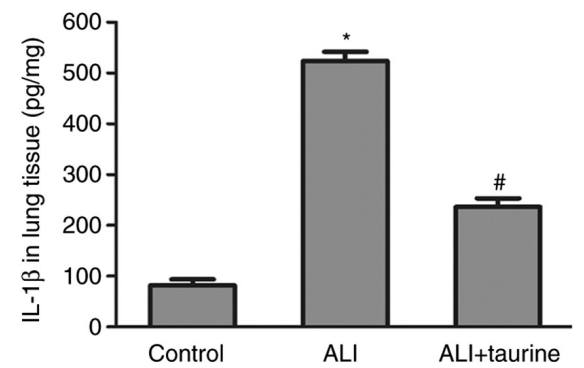

B

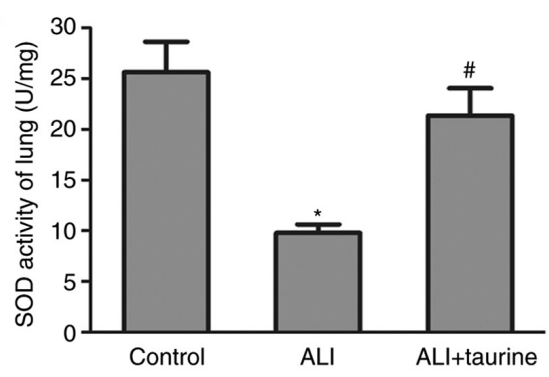

D

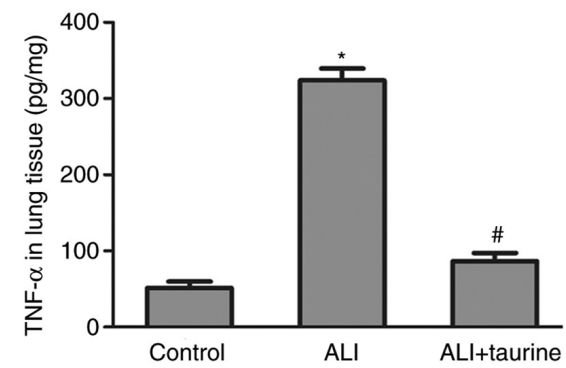

Figure 2. Taurine attenuates oxidative stress and the inflammatory response. To assess the protective effects of taurine on oxidative stress and the inflammatory response, MDA levels, SOD activity, and TNF- $\alpha$ and IL-1 $\beta$ concentrations in the lung tissues of septic rats were detected. (A) MDA levels and (B) SOD activity in the lung tissues of each group. (C) TNF- $\alpha$ and (D) IL-1 $\beta$ concentrations in the lung tissues of each group. Data are presented as the mean \pm standard deviation. ${ }^{*} \mathrm{P}<0.05$ vs. control; " $\mathrm{P}<0.05$ vs. ALI. MDA, malondiadelhyde; SOD, superoxide dismutase; ALI, acute lung injury.
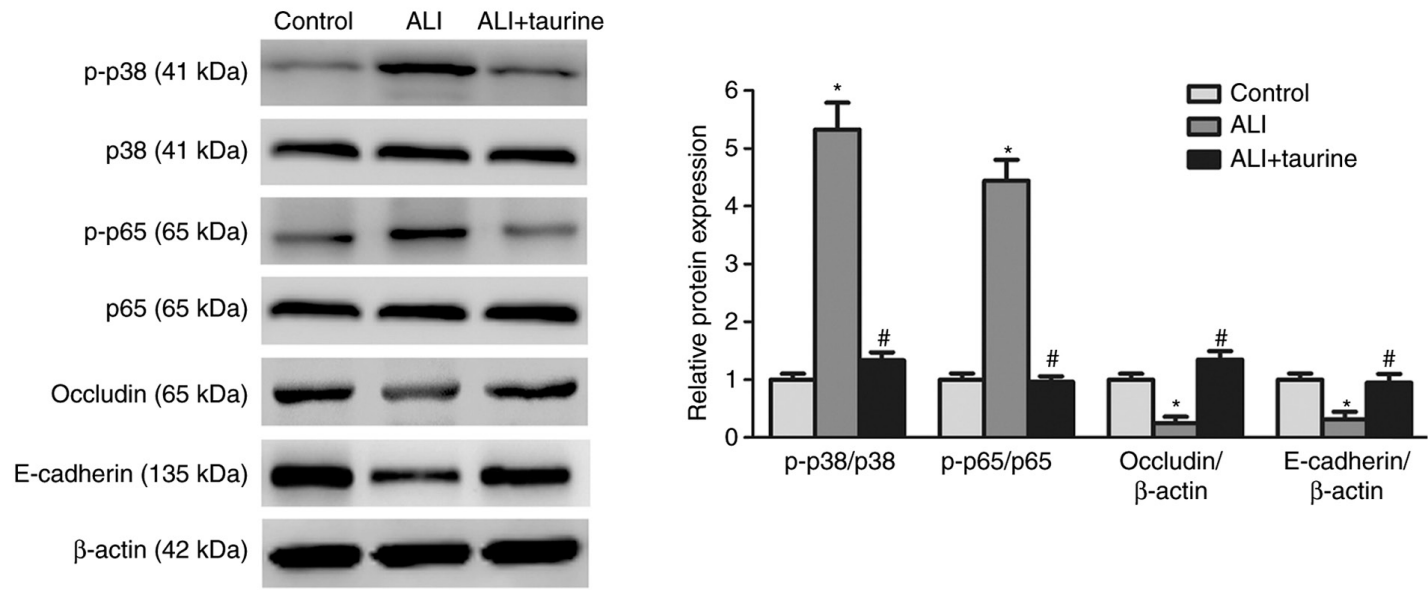

Figure 3. Effects of taurine on the expression levels of p38/MAPK and NF-кB signaling pathway-related proteins and epithelial markers. Protein expression levels of E-cadherin, occludin, p-p38/p38 and p-p65/p65 in the lung tissues of cecal ligation and puncture-induced septic ALI model rats were determined by western blotting and semi-quantified. "P<0.05 vs. control; "P<0.05 vs. ALI. p, phosphorylated; ALI, acute lung injury.

Taurine downregulates TNF- $\alpha$ and IL-1 $\beta$ concentrations in lung tissue. The inflammatory response is induced in sepsis (20). Therefore, at 3 days post-CLP, IL- $1 \beta$ and TNF- $\alpha$ concentrations in the lung tissues of septic rats were measured by performing ELISAs. The results demonstrated that the concentrations of IL-1 $\beta$ and TNF- $\alpha$ were significantly elevated in the lung tissues of CLP-induced septic ALI model rats compared with those in the control group (Fig. 2C and D). However, the concentrations of IL- 6 and TNF- $\alpha$ were significantly decreased in the taurine treatment group compared with those in the ALI group. The results indicated that taurine treatment inhibited the inflammatory response in sepsis-induced lung injury.

Taurine downregulates the expression levels of $38 / M A P K$ and $N F-\kappa B$ signaling pathway-related proteins. The p38/MAPK and $\mathrm{NF}-\kappa \mathrm{B}$ signaling pathways are key upstream pathways in the sepsis-induced inflammatory response (30). To further investigate the molecular mechanisms underlying the anti-inflammatory effects of taurine, whether taurine inhibited sepsis-induced activation of the p38/MAPK and $N F-\kappa B$ signaling pathways was investigated. Western blotting was performed to detect the protein expression levels of p-p38 and p-p65, which are key proteins of the p38/MAPK and NF- $\kappa$ B signaling pathways (30), respectively. Compared with those in the control group, the ratios of p-p38/p38 and p-p65/p65 were significantly increased in the lung tissues of septic rats, indicating that the $\mathrm{p} 38 / \mathrm{MAPK}$ and NF- $\mathrm{B}$ signaling pathways were activated in the injured lung tissues (Fig. 3). However, the protein expression levels of the epithelial markers, E-cadherin and occludin, were significantly decreased in the lung tissues 

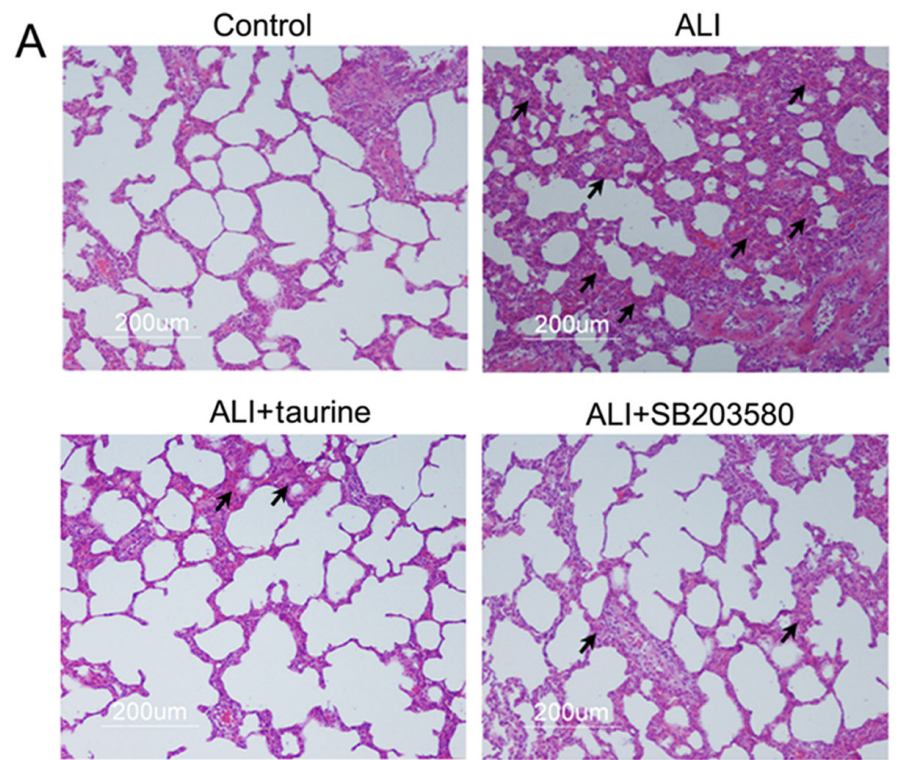

B

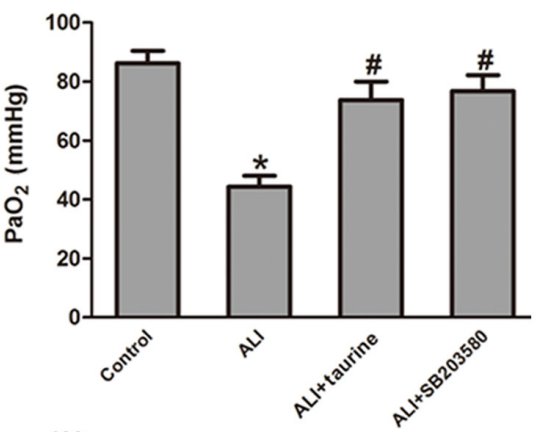

ALI+SB203580

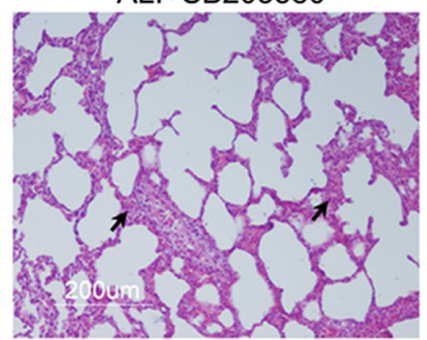

C

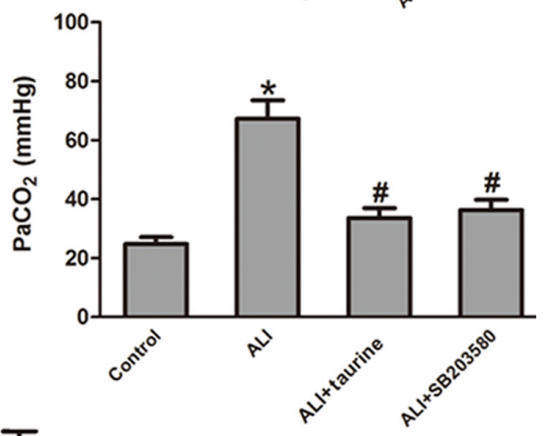

E

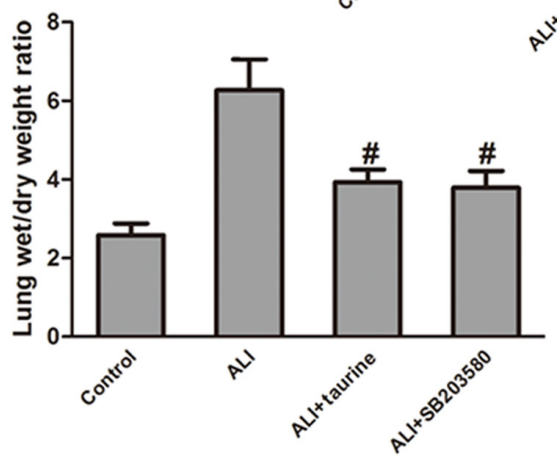

Figure 4. SB203580 protects against sepsis-induced ALI. SB203580, an inhibitor of p38/MAPK signaling, was used to treat septic rats to assess the role of the p38/MAPK signaling pathway in the pathogenesis of sepsis-induced ALI. SB203580 ameliorated lung injury and promoted lung repair by blocking p38/MAPK signaling pathway. The results displayed no significant difference between the SB203580 and taurine treatment groups. (A) Representative images of hematoxylin and eosin staining in each group (magnification, x100). Black arrows indicate neutrophil infiltration. Content of (B) $\mathrm{O}_{2}$ and $(\mathrm{C}) \mathrm{CO}_{2}$ in arterial blood (D) Lung injury score in each group. (E) Lung wet/dry weight ratio of the lung tissues in each group. Data are presented as the mean \pm standard deviation. ${ }^{*} \mathrm{P}<0.05$ vs. control; ${ }^{*} \mathrm{P}<0.05$ vs. ALI. ALI, acute lung injury; Pa, partial pressure.

of CLP-induced septic ALI model rats compared with those in the control group. By contrast, taurine significantly decreased the protein expression levels of p-p38 and p-p65 and increased the expression levels of E-cadherin and occludin in the ALI group. Collectively, these results suggested that taurine inhibited sepsis-induced lung injury and protected lung epithelium by blocking the p38/MAPK and NF- $\mathrm{KB}$ signaling pathways, which were activated by sepsis.

p38/MAPK inhibitor SB203580 attenuates sepsis-induced lung injury. To identify the functions of the p38/MAPK signaling pathway in sepsis-induced ALI, CLP-induced septic ALI model rats were treated with or without the p38/MAPK inhibitor SB203580. The H\&E staining results demonstrated that SB203580 markedly attenuated lung injury in CLP-induced septic ALI model rats treated with SB203580 compared with the ALI group (Fig. 4A). SB203580 treatment significantly increased $\mathrm{PaO}_{2}$ levels and decreased $\mathrm{PaCO}_{2}$ levels in the arterial blood of CLP-induced septic ALI model rats compared with those in the ALI group (Fig. 4B and C), which suggested that SB203580 suppressed sepsis-induced lung functions, a similar effect to that observed in the taurine treatment group.
Furthermore, the lung injury score and lung wet/dry ratio were determined in each group. As shown in Fig. 4D and E, the results indicated that SB203580 significantly decreased the lung injury score and lung wet/dry ratio in CLP-induced septic ALI model rats compared with those in the ALI group. Notably, the results of SB203580 treatment group were similar to those in the taurine treatment group, which suggested that the p38/MAPK signaling pathway may serve as an effective therapeutic target for sepsis-induced ALI.

SB203580 suppresses oxidative stress and the inflammatory response. To determine the effects of SB203580 on oxidative stress and the inflammatory response, MDA and SOD expression levels and IL- $1 \beta$ and TNF- $\alpha$ concentrations in the lung tissues of each group were detected. The results demonstrated that SB203580 treatment significantly decreased MDA levels and the concentrations of IL- $1 \beta$ and TNF- $\alpha$, but restored SOD activities compared with those in the ALI group (Fig. 5). The results in the ALI + SB203580 group were similar to those in the ALI + taurine group, which suggested that SB203580 may protect against CLP-induced lung injury by inhibiting the p38/MAPK signaling pathway. The results demonstrated that 
A

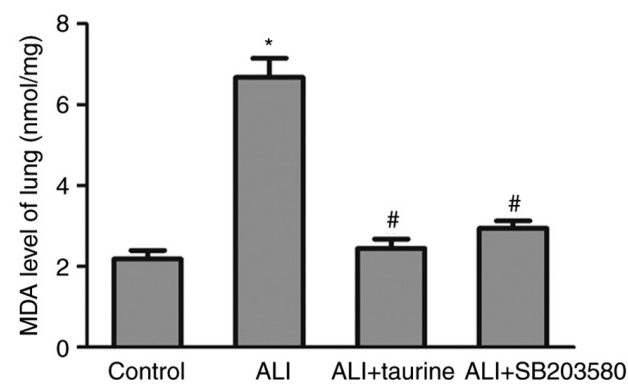

C

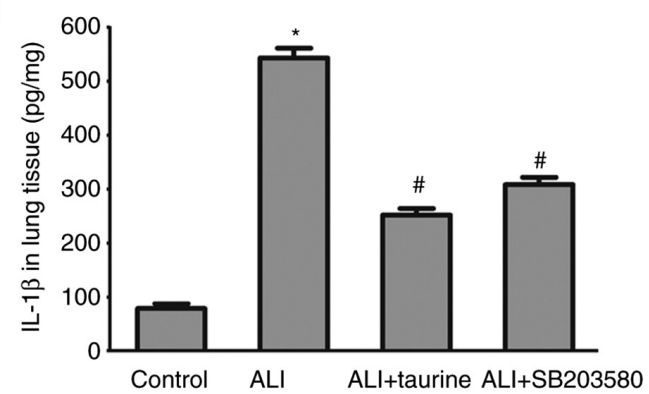

B

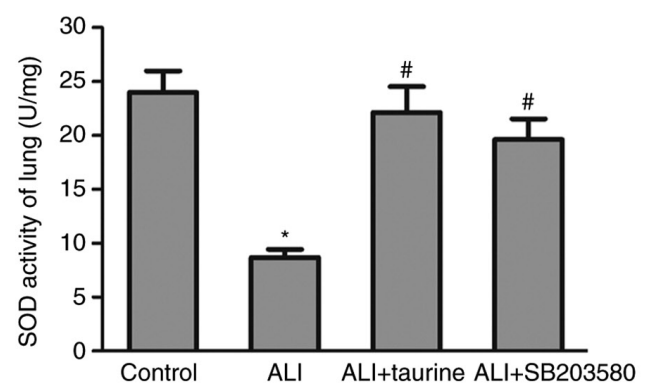

D

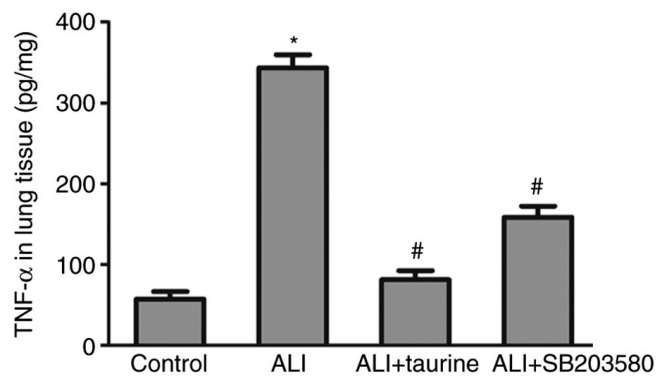

Figure 5. p38/MAPK signaling suppression inhibits sepsis-induced oxidative stress and inflammatory responses in cecal ligation and puncture-induced septic ALI model rats. SB203580 attenuated MDA levels, SOD activity, and TNF- $\alpha$ and IL-1 $\beta$ concentrations in the lung tissues of septic rats. (A) MDA levels and (B) SOD activity in the lung tissues of each group. (C) TNF- $\alpha$ and (D) IL-1 $\beta$ concentrations in the lung tissues of each group. Data are presented as mean \pm standard deviation. "P<0.05 vs. control; "P<0.05 vs. ALI. ALI, acute lung injury; MDA, malondiadelhyde; SOD, superoxide dismutase.
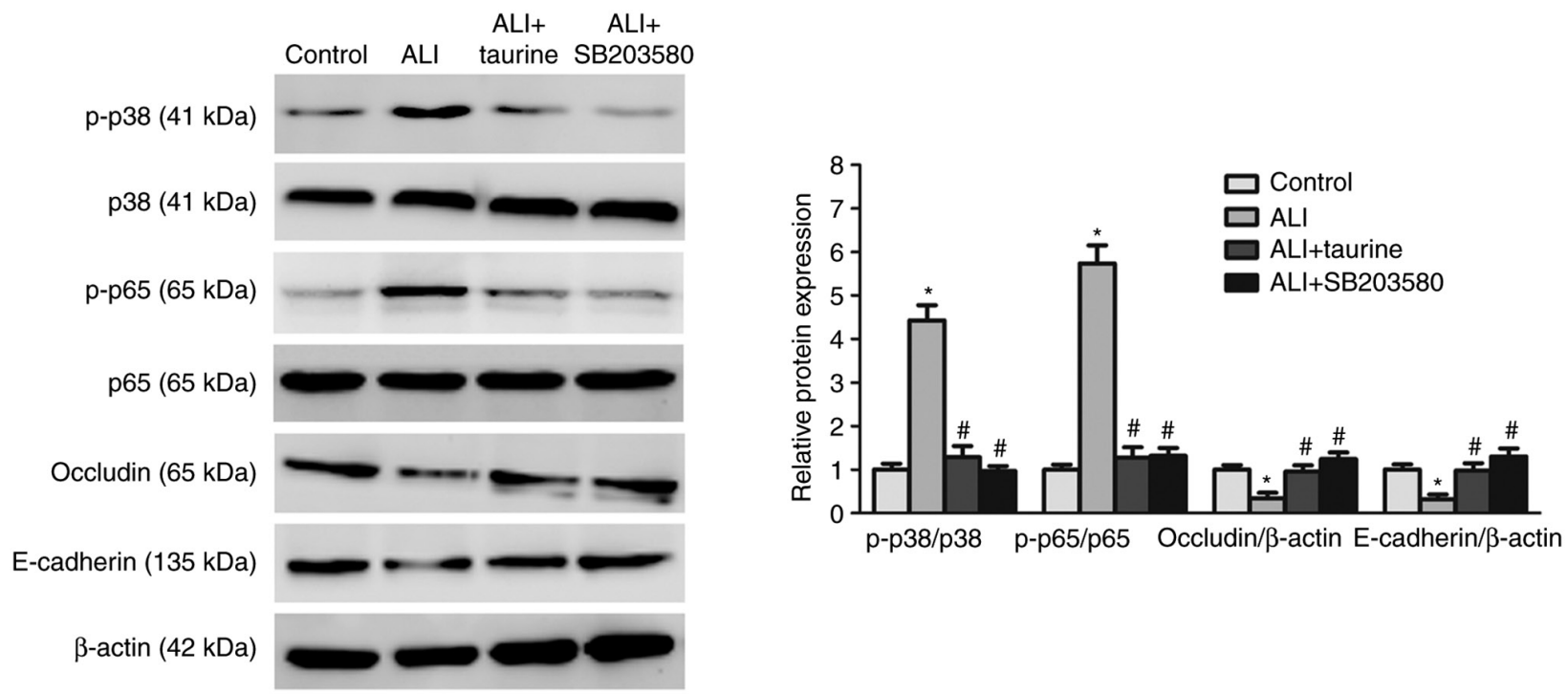

Figure 6. SB203580 inhibits the p38/MAPK signaling pathway to promote lung repair. Protein expression levels of E-cadherin, occludin, p-p38/p38 and p-p65/p65 in the lung tissues of CLP-induced septic ALI model rats following treatment with SB20380 were determined by western blotting and semi-quantified. SB20380 treatment significantly increased the expression levels of E-cadherin and occludin, but significantly decreased the ratios of p-p38/p38 and p-p65/p65 in ALI rats. ${ }^{*} \mathrm{P}<0.05$ vs. control; ${ }^{*} \mathrm{P}<0.05$ vs. ALI. p, phosphorylated; ALI, acute lung injury.

the p38/MAPK signaling pathway may serve an essential role in the development of sepsis-induced ALI.

SB203580 inhibits sepsis-activated p38/MAPK signaling. The present study further indicated that treatment with SB203580 significantly reduced the ratio of p-p38/p38 in septic rats, suggesting that SB203580 inhibited activation of the p38/MAPK signaling pathway mediated by CLP-induced sepsis (Fig. 6). In addition, the western blotting results demonstrated that the protein expression levels of E-cadherin and occludin were significantly increased in the ALI + taurine group compared with those in the ALI group. The results of the ALI + SB203580 group were also similar to those in the ALI + taurine group, indicating that SB203580 protected lung epithelium against sepsis-induced damage, which was similar to the protective effects of taurine. Furthermore, it was observed that the ratio of p-p65/p65 was also significantly decreased in the lung tissues of CLP-induced septic ALI model rats treated with SB203580 compared with those in the ALI group, which suggested that 38 /MAPK may be an upstream of 
the $N F-\kappa B$ signaling pathway. The results indicated that the suppressed p38 MAPK signaling pathway was associated with the expression level of $\mathrm{NF}-\kappa \mathrm{B}$ signaling-related proteins in CLP-induced septic ALI model rats treated with SB203580, which was similar to the taurine treatment group. Collectively, the results indicated that taurine may serve a protective role in lung epithelium damage in septic rats via modulating the p38 MAPK signaling pathway.

\section{Discussion}

Sepsis, as one of the leading causes of death in patients who are critically ill, induces a complex systemic inflammatory response syndrome and subsequent multiple organ failure (31,32). Multiple organ dysfunction syndromes, particularly ALI/ARDS, are major causes of morbidity and mortality in patients with sepsis (33). ALI/ARDS, which is induced by sepsis, is characterized by the rapid onset of respiratory insufficiency, acute inflammatory response, alveolar epithelium damage, bleeding and lung interstitial edema $(6,34,35)$. The pathogenesis of ALI/ARDS is not completely understood and there are no specific effective therapeutic strategies available $(36,37)$. Therefore, it is important to investigate the molecular mechanisms underlying sepsis-induced ALI/ARDS to develop effective treatments.

Taurine, an endogenous free amino acid, is highly expressed in numerous mammalian tissues. Taurine has been reported to be an effective cell protector, which could protect against lung damage by mitigating oxidative stress and reducing the expression of inflammatory factors $(20,21)$. Previously, Li et al (21) reported that taurine could reverse paraquat-induced disturbances in glutathione (GSH) content and GSH peroxidase activity. Moreover, taurine attenuated paraquat-induced A549 cell apoptosis via modulation of oxidative stress. Although it has been reported that taurine reverses paraquat-induced oxidative stress to protect against cell apoptosis, the mechanisms underlying taurine-mediated regulation of oxidative stress remain unclear. In contrast to the CLP-induced septic ALI rat model established in the present study, Men et al (26) demonstrated that taurine treatment attenuated lung injury following limb ischemia reperfusion (LIR). It has also been reported that taurine prevents oxidative stress-induced cell apoptosis, decreases MDA level, and increases SOD and catalase activities by inhibiting endoplasmic reticulum stress. However, whether taurine attenuates the expression levels of inflammatory factors to protect against LIR-induced lung injury is not completely understood. Our previous study indicated that taurine enhanced the protective effect of dexmedetomidine on sepsis-induced lung injury by suppressing activation of the $\mathrm{NF}-\kappa \mathrm{B}$ signaling pathway and decreased the expression levels of IL- 6 and IL-1 $\beta$ (20). Taurine serves an essential role in regulating the $\mathrm{T}$ helper $(\mathrm{Th}) 1 / \mathrm{Th} 2$ cytokine balance (38). In the present study, the protective effects of taurine on sepsis-induced lung injury were investigated. Taurine treatment preserved epithelium integrity by increasing the expression levels of epithelium markers E-cadherin and occludin to alleviate CLP-induced lung injury in rats. The proinflammatory cytokines TNF- $\alpha$ and IL-1 $\beta$ are implicated in the pathogenesis of sepsis-induced lung injury (39). The present study demonstrated that taurine treatment decreased sepsis-induced increases in TNF- $\alpha$ and IL-1 $\beta$ expression levels in lung tissues, indicating that taurine treatment inhibited the inflammatory response in CLP-induced septic ALI model rats. Oxidative stress serves an essential role in the pathogenesis of ALI (40). Therefore, oxidative stress levels were assessed in the present study by detecting MDA levels and SOD activities. MDA levels are an index of membrane lipid peroxidation and can be used as a marker to indicate the degree of tissue peroxidation damage. SOD serves an important role in the balance of oxidation and antioxidation (25). The results of the present study indicated that taurine effectively attenuated oxidative stress damage in sepsis-induced lung injury, suggesting that taurine could be used as a protective factor to attenuate lung injury by decreasing sepsis-induced inflammatory responses and oxidative stress. Moreover, taurine may protect epithelium integrity by promoting the expression of epithelial markers. Therefore, the present study further suggested that taurine may serve an essential role in the lung repair process.

To clarify the mechanisms underlying taurine-mediated protection of lung tissues after CLP induction, p38/MAPK and $\mathrm{NF}-\kappa \mathrm{B}$ signaling pathways involved in sepsis-induced inflammatory responses and oxidative stress were investigated. Recent studies have demonstrated that the p38/MAPK signaling pathway is one of the most important signaling pathways, serving a critical role in the phosphorylation of substrates involved in the sepsis-induced inflammatory response $(12,39)$. Certain studies have indicated that the p38/MAPK signaling pathway also serves an essential role in regulating oxidative stress-related signaling and participates in the pathogenesis of ALI $(12,41,42)$. It is important to identify the changes in $338 /$ MAPK activity associated with sepsis-induced ALI; therefore, the protein expression levels of p-p38 in the p38/MAPK signaling pathway were detected in the lung tissues of CLP-induced septic ALI model rats. The results demonstrated that taurine suppressed sepsis-induced activation of the p38/MAPK signaling pathway. Furthermore, the $N F-\kappa B$ signaling pathway, which was previously reported to be involved in the regulation of inflammatory mediator generation and immune responses $(43,44)$, was also investigated in CLP-induced septic ALI model rats. The results demonstrated that taurine treatment decreased the ratio of p-p65/p65 to block NF- $\kappa$ B signaling in CLP-induced septic ALI model rats. To investigate the role of the p38/MAPK signaling pathway in the pathogenesis of sepsis-induced lung injury, SB203580, an inhibitor of the p38/MAPK signaling pathway, was used to treat the CLP-induced septic ALI model rats. In the present study, the $\mathrm{H} \& \mathrm{E}$ staining and respiratory function test results indicated that SB203580 attenuated lung injury, reduced inflammatory cell infiltration and promoted lung restoration in CLP-induced septic ALI model rats. Furthermore, MDA levels and SOD activity, which are important indexes of oxidative stress, were significantly decreased after SB203580 treatment in CLP-induced septic ALI model rats, which indicated that inhibition of the p38/MAPK signaling pathway could ameliorate oxidative stress. The concentrations of proinflammatory cytokines, TNF- $\alpha$ and IL-1 $\beta$, were significantly decreased in the SB203580 treatment group compared with those in the ALI group, suggesting that SB203580 administration reduced local inflammation in CLP-induced septic ALI model rats. Moreover, sepsis-induced p38/MAPK 
phosphorylation was attenuated by treatment with SB203580. In addition, SB203580 administration also decreased the ratio of p-p65/p65, a NF- $\kappa$ B signaling pathway-related protein, in CLP-induced septic ALI model rats. Similarly, the results indicated that inhibition of $\mathrm{p} 38 / \mathrm{MAPK}$ signaling could restore the expression levels of lung epithelium markers, suggesting that SB203580 protected against sepsis-induced lung epithelium damage. The results observed following SB203580 treatment of septic rats were similar to those observed in the taurine treatment group, which suggested that taurine attenuated lung injury and promoted lung repair by blocking the p38/MAPK signaling pathway. The present study also revealed that inhibition of the p38/MAPK signaling pathway could alter the activity of the NF- $\mathrm{B}$ signaling pathway, which suggested that the p38/MAPK signaling pathway may crosstalk with the $N F-\kappa B$ signaling pathway for the generation of inflammatory factors and the immune response in sepsis-induced ALI. However, the present study only used the CLP-induced septic ALI rat model to investigate the protective effects and molecular mechanisms underlying taurine in sepsis-induced lung injury. Due to this limitation, a lung epithelial cell line should be treated with LPS to establish an in vitro experiment model to verify the results of the present study and investigate the role of $\mathrm{p} 38 / \mathrm{MAPK}$ signaling or downstream signaling of p38/MAPK in regulating the inflammatory response and oxidative stress during the pathogenesis of LPS-induced lung epithelium damage.

In conclusion, to the best of our knowledge, the present study was the first to investigate the protective effects of taurine on sepsis-induced lung injury via suppressing the p38/MAPK signaling pathway, inhibiting the inflammatory response and oxidative stress levels, and preserving lung epithelium integrity. The present study demonstrated that taurine displayed potential therapeutic effects on sepsis-induced lung injury, and the $\mathrm{p} 38 / \mathrm{MAPK}$ signaling pathway may serve as a promising therapeutic target for sepsis-induced lung injury.

\section{Acknowledgements}

The authors would like to thank Dr Zhaorui Sun (Jinling Hospital, Nanjing, China) for their technical assistance.

\section{Funding}

The present study was supported by the Science and Technology Development Foundation of Nanjing Medical University (grant no. NMUB2018288).

\section{Availability of data and materials}

The datasets used and/or analyzed during the current study are available from the corresponding author on reasonable request.

\section{Authors' contributions}

JCh and XX performed the majority of the experiments. JCa and $\mathrm{LJ}$ performed the statistical analyses and performed some of the experiments. BS and WZ designed the study and analyzed the data. All authors contributed to preparing the manuscript. All authors have read and approved the final manuscript. BS and $\mathrm{WZ}$ confirm the authenticity of all the raw data.

\section{Ethics approval and consent to participate}

All animal studies were conducted in accordance with the Guide for the Care and Use of Experimental Animals established by National Society for Medical Research and approved by the Ethics Committee of Nanjing Medical University (approval no. SYXK 2019-0042).

\section{Patient consent for publication}

Not applicable.

\section{Competing interests}

The authors declare that they have no competing interests.

\section{References}

1. Kumar V: Pulmonary Innate Immune Response Determines the Outcome of Inflammation During Pneumonia and Sepsis-Associated Acute Lung Injury. Front Immunol 11: 1722, 2020.

2. Lelubre C and Vincent JL: Mechanisms and treatment of organ failure in sepsis. Nat Rev Nephrol 14: 417-427, 2018.

3. Singer M, Deutschman CS, Seymour CW, Shankar-Hari M, Annane D, Bauer M, Bellomo R, Bernard GR, Chiche JD, Coopersmith CM, et al: The Third International Consensus Definitions for Sepsis and Septic Shock (Sepsis-3). JAMA 315: 801-810. 2016.

4. Fang WF, Huang CH, Chen YM, Hung KY, Chang YC, Lin CY, Fang YT, Chang YT, Chen HC, Huang KT, et al: Application of dynamic pulse pressure and vasopressor tools for predicting outcomes in patients with sepsis in intensive care units. J Crit Care 52: 156-162, 2019.

5. Sterling SA, Puskarich MA, Glass AF, Guirgis F and Jones AE The Impact of the Sepsis-3 Septic Shock Definition on Previously Defined Septic Shock Patients. Crit Care Med 45: 1436-1442, 2017.

6. Li T and Zou C: The Role of Deubiquitinating Enzymes in Acute Lung Injury and Acute Respiratory Distress Syndrome. Int J Mol Sci 21: 4842, 2020.

7. Fleischmann C, Scherag A, Adhikari NK, Hartog CS, Tsaganos T, Schlattmann P, Angus DC and Reinhart K; International Forum of Acute Care Trialists; Current Estimates and Limitations: Assessment of Global Incidence and Mortality of Hospital-treated Sepsis. Am J Respir Crit Care Med 193: 259-272, 2016.

8. Yang L, Liu S, Han S, Hu Y, Wu Z, Shi X, Pang B, Ma Y and Jin J: The HDL from septic-ARDS patients with composition changes exacerbates pulmonary endothelial dysfunction and acute lung injury induced by cecal ligation and puncture (CLP) in mice. Respir Res 21: 293, 2020.

9. Qiu X, Liang X, Li H and Sun R: LPS-induced vein endothelial cell injury and acute lung injury have Btk and Orai 1 to regulate SOC-mediated calcium influx. Int Immunopharmacol 90: 107039, 2021

10. Li L, Dong L, Zhao D, Gao F and Yan J: Classical dendritic cells regulate acute lung inflammation and injury in mice with lipopolysaccharide induced acute respiratory distress syndrome. Int J Mol Med 44: 617-629, 2019.

11. Wang F, Wang M, Wang J, Chen M, Sun S, Yao S and Xia H: Maresin 1 ameliorates sepsis-associated lung injury by inhibiting the activation of the JAK2/STAT3 and MAPK/ NF- $\kappa$ B signaling pathways. Microb Pathog 148: 104468, 2020.

12. Hu XH, Situ HL, Chen JP and Yu RH: Lipoxin A4 alleviates lung injury in sepsis rats through p38/MAPK signaling pathway. J Biol Reg Homeost Agents 34: 807-814, 2020.

13. Zhang HF, Zhang HB, Wu XP, Guo YL, Cheng WD and Qian F: Fisetin alleviates sepsis-induced multiple organ dysfunction in mice via inhibiting p38 MAPK/MK2 signaling. Acta Pharmacol Sin 41: 1348-1356, 2020. 
14. Shao Y, Chen X, Liu Y, Chen X, Li C, Wang L and Zhao W: Dexmedetomidine alleviates lung injury in sepsis mice through regulating P38 MAPK signaling pathway. Panminerva Med: Mar 30, 2020 (Epub ahead of print). doi: 10.23736/S0031-0808.20.03885-9.

15. Feng Y, Fang Z, Liu B and Zheng X: p38MAPK plays a pivotal role in the development of acute respiratory distress syndrome. Clinics (São Paulo) 74: e509, 2019.

16. Chen X, Hu J, Pan Y and Tang Z: Novel noncoding RNAs biomarkers in acute respiratory distress syndrome. Expert Rev Respir Med 14: 299-306, 2020

17. Jakaria M, Azam S, Haque ME, Jo SH, Uddin MS, Kim IS and Choi DK: Taurine and its analogs in neurological disorders: Focus on therapeutic potential and molecular mechanisms. Redox Biol 24: 101223, 2019.

18. Schaffer S and Kim HW: Effects and Mechanisms of Taurine as a Therapeutic Agent. Biomol Ther (Seoul) 26: 225-241, 2018.

19. Thirupathi A, Pinho RA, Baker JS, István B and Gu Y: Taurine Reverses Oxidative Damages and Restores the Muscle Function in Overuse of Exercised Muscle. Front Physiol 11: 582449, 2020

20. Zhao W, JiaL, Yang HJ, Xue X, Xu WX, Cai JQ, Guo RJ and Cao CC: Taurine enhances the protective effect of Dexmedetomidine on sepsis-induced acute lung injury via balancing the immunological system. Biomed Pharmacother 103: 1362-1368. 2018.

21. Li S, Wang J, Wei BK, Dong G and Wang X: Protective Effect of Taurine on Paraquat-Induced Lung Epithelial Cell Injury. Adv Exp Med Biol 1155: 739-746, 2019.

22. Gao Y, Li X, Gao J, Zhang Z, Feng Y, Nie J, Zhu W, Zhang S and Cao J: Metabolomic Analysis of Radiation-Induced Lung Injury in Rats: The Potential Radioprotective Role of Taurine. Dose Response 17: 1559325819883479, 2019.

23. Sun Z, Yang Z, Wang M, Huang C, Ren Y, Zhang W, Gao F, Cao L, Li L and Nie S: Paraquat induces pulmonary fibrosis through $\mathrm{Wnt} / \beta$-catenin signaling pathway and myofibroblast differentiation. Toxicol Lett 333: 170-183, 2020.

24. Chen Z, Ding X, Jin S, Pitt B, Zhang L, Billiar T and Li Q: WISP1- $\alpha v \beta 3$ integrin signaling positively regulates TLR-triggered inflammation response in sepsis induced lung injury. Sci Rep 6: 28841, 2016.

25. Liu Q, Hua F, Deng C, Zhang J, Xu G and Hu Y: Protective and therapeutic effects of Danhong injection on acute pancreatitis associated lung injury. Mol Med Rep 16: 7603-7608, 2017.

26. Men X, Han S, Gao J, Cao G, Zhang L, Yu H, Lu H and Pu J: Taurine protects against lung damage following limb ischemia reperfusion in the rat by attenuating endoplasmic reticulum stress-induced apoptosis. Acta Orthop 81: 263-267, 2010.

27. Tanino Y, Makita H, Miyamoto K, Betsuyaku T, Ohtsuka Y, Nishihira $J$ and Nishimura $M$ : Role of macrophage migration inhibitory factor in bleomycin-induced lung injury and fibrosis in mice. Am J Physiol Lung Cell Mol Physiol 283: L156-L162, 2002.

28. Sun Z, Gong X, Zhu H, Wang C, Xu X, Cui D, Qian W and Han X: Inhibition of Wnt $/ \beta$-catenin signaling promotes engraftment of mesenchymal stem cells to repair lung injury. J Cell Physiol 229 213-224, 2014

29. Park I, Kim M, Choe K, Song E, Seo H, Hwang Y, Ahn J, Lee SH, Lee JH, Jo YH, et al: Neutrophils disturb pulmonary microcirculation in sepsis-induced acute lung injury. Eur Respir J 53: 53 2019.

30. Cong Z, Li D, Tao Y, Lv X and Zhu X: $\alpha 2 \mathrm{~A}$-AR antagonism by BRL-44408 maleate attenuates acute lung injury in rats with downregulation of ERK1/2, p38MAPK, and p65 pathway. J Cell Physiol 235: 6905-6914, 2020.
31. Rhodes A, Phillips G, Beale R, Cecconi M, Chiche JD, De Backer D, Divatia J, Du B, Evans L, Ferrer R, et al: The Surviving Sepsis Campaign bundles and outcome: results from the International Multicentre Prevalence Study on Sepsis (the IMPreSS study). Intens Care Med 41: 1620-1628. 2015.

32. Machado FR, Cavalcanti AB, Bozza FA, Ferreira EM, Angotti Carrara FS, Sousa JL, Caixeta N, Salomao R, Angus DC, Pontes Azevedo LC, et al; SPREAD Investigators; Latin American Sepsis Institute Network: The epidemiology of sepsis in Brazilian intensive care units (the Sepsis PREvalence Assessment Database, SPREAD): An observational study. Lancet Infect Dis 17: 1180-1189, 2017.

33. Fan EKY and Fan J: Regulation of alveolar macrophage death in acute lung inflammation. Respir Res 19: 50, 2018.

34. Magnani ND, Dada LA and Sznajder JI: Ubiquitin-proteasome signaling in lung injury. Transl Res 198: 29-39, 2018.

35. Matthay MA, Arabi YM, Siegel ER, Ware LB, Bos LDJ, Sinha P, Beitler JR, Wick KD, Curley MAQ, Constantin JM, et al: phenotypes and personalized medicine in the acute respiratory distress syndrome. Intensive Care Med 46: 2136-2152, 2020.

36. Masterson C, Jerkic M, Curley GF and Laffey JG: Mesenchymal stromal cell therapies: Potential and pitfalls for ARDS. Minerva Anestesiol 81: 179-194, 2015

37. Wang YM, Qi X, Gong FC, Chen Y, Yang ZT, Mao EQ and Chen EZ: Protective and predictive role of Mucin1 in sepsis-induced ALI/ARDS. Int Immunopharmacol 83: 106438, 2020.

38. Lu YF, Zhang QY, Wang LH, Liu XY and Zhang SX: The protective effects of taurine on experimental autoimmune myocarditis. Eur Rev Med Pharm 21: 1868-1875, 2017.

39. Shao Z, Li Q, Wang S and Chen Z: Protective effects of PNU 282987 on sepsis induced acute lung injury in mice. Mol Med Rep 19: 3791-3798, 2019.

40. Bhavsar TM, Cantor JO, Patel SN and Lau-Cam CA: Attenuating effect of taurine on lipopolysaccharide-induced acute lung injury in hamsters. Pharmacol Res 60: 418-428, 2009.

41. Cui S, Nian Q, Chen G, Wang X, Zhang J, Qiu J and Zhang Z: Ghrelin ameliorates A549 cell apoptosis caused by paraquat via $\mathrm{p} 38$-MAPK regulated mitochondrial apoptotic pathway. Toxicology 426: 152267, 2019.

42. Zhang CY, Lin SQ, Liu FY, Ma JH, Jia FJ, Han Z, Xie WD and Li X: The anti-inflammatory effect of -kaur-15-en-17-al-18-oic acid on lipopolysaccharide-stimulated RAW264.7 cells associated with NF- $\mathrm{BB}$ and P38/MAPK pathways. J Asian Nat Prod Res 23: 570-583, 2021.

43. Fang H, Zhang J, Ao M, He F, Chen W, Qian Y, Zhang Y, $\mathrm{Xu} \mathrm{Y}$ and Fang M: Synthesis and discovery of $\omega-3$ polyunsaturated fatty acid- alkanolamine (PUFA-AA) derivatives as anti-inflammatory agents targeting Nur77. Bioorg Chem 105: 104456, 2020.

44. Xiao Q, Cui Y, Zhao Y, Liu L, Wang H and Yang L: Orientin relieves lipopolysaccharide-induced acute lung injury in mice: The involvement of its anti-inflammatory and anti-oxidant properties. Int Immunopharmacol 90: 107189, 2021.

This work is licensed under a Creative Commons Attribution-NonCommercial-NoDerivatives 4.0 International (CC BY-NC-ND 4.0) License. 